Classification

Physics Abstracts

$68.45-81.60-82.70$

\title{
Scaling theory of polymer adsorption : proximal exponent
}

\author{
P. G. de Gennes \\ Collège de France, 75231 Paris Cedex 05, France \\ and P. Pincus (*) \\ Institute for Theoretical Physics, UC Santa Barbara, Santa Barbara, Ca., U.S.A.
}

(Reçu le 19 janvier 1983, accepté le 3 février 1983)

\begin{abstract}
Résumé. - Nous interprétons des calculs récents de Eisenriegler, Kremer et Binder [1], et de Diehl et Dietrich [2]. Nous concluons que le profil de concentration $\phi(x)$ (pour une chaîne isolée, en bon solvant) est singulier près du múr $\phi(x) \sim(a / x)^{m}$ où $m \cong 1 / 3$. Prenant en compte l'exposant proximal $m$, nous corrigeons certaines lois d'échelle proposées antérieurement $[3,4]$ pour la tension interfaciale de solutions semi-diluées en bon solvant. Nous trouvons aussi que l'exposant proximal est en bon accord avec un calcul de séries dû à Ishinabe [5] sur l'énergie d'une chaîne isolée.
\end{abstract}

\begin{abstract}
We interpret some recent calculations of Eisenriegler, Kremer, and Binder [1], and related multi-critical results of Diehl and Dietrich [2]. They show that the monomer concentration profile $\phi(x)$ for isolated polymer chains in a good solvent and in close proximity to an adsorbing wall exhibits a singular behaviour; i.e., $\phi(x) \sim(a / x)^{m}$, where $a$ is a monomer dimension and $m \cong 1 / 3$. Taking into account the proximal exponent $m$, we correct earlier scaling results $[3,4]$ for the interfacial tension of semi-dilute polymer solutions in good solvents. We also find good agreement with a recent result by Ishinabe [5] on the single chain energy.
\end{abstract}

\section{Introduction.}

In a previous scaling theory [3] of interfacial properties of semi-dilute polymer solutions (in good solvents) against an adsorbing surface, the monomer concentration profile $\phi(x)$ was described in terms of three spatial regions : 1$)$ the proximal region $x<D(D$ is approximately the thickness of an isolated adsorbed polymer chain) where $\phi(x)$ was assumed to be slowly varying (approaching the surface value $\phi_{\mathrm{s}}$ at $\left.\left.x=0\right) ; 2\right)$ the central region $\xi_{\mathrm{b}}>x>D\left(\xi_{\mathrm{b}}\right.$ is the bulk correlation length [6]), where $\phi(x)$ is self-similar and decays as a power law ; 3 ) the distal region $x>\xi_{\mathrm{b}}$ where $\phi(x)$ approaches $\phi_{\mathrm{b}}$ exponentially. An essential assumption in this analysis was that in the proximal region the profile is not singular. A renormalization group calculation to first order [7] in $\varepsilon$ ( $\varepsilon=4-d$, where $d$ is the space dimension) is in accord with this conjecture.

(*) Permanent address : Exxon Research, P. O. Box 45, Linden, NJ 07036, U.S.A. 
However, more recently, Diehl and Dietrich [2] have studied general interfacial problems to second order in $\varepsilon$. Also Eisenriegler et al. [1] have carried out Monte Carlo calculations for a single chain near an adsorbing wall. The resulting proximal behaviour is quite different, and will be presented in section 2 . In section 3 we propose an extension of these results to many chain adsorption.

\section{The single chain problem.}

Consider our flexible coil with $N$ monomers, each of size $a$, dissolved in a good solvent, and weakly adsorbed on a flat wall. In terms of a lattice model (with mesh size $a$ ) each monomer on the first layer (at a distance $x=a$ from the wall) feels an attractive energy $-k T \delta$ (where $\delta$ is small). The thickness $D$ of the adsorbed coil (measured normal to the wall) has the scaling structure

$$
D=a \delta^{-u} w\left(N^{\phi} \delta\right)=a \delta^{-u} w(x),
$$

where $\phi$ is called a crossover exponent $\left({ }^{1}\right)$, and $u$ is another exponent to be defined below. The function $w(x)$ has the following limits

$$
\begin{aligned}
& w(x \rightarrow \infty)=1, \\
& w(x \rightarrow 0)=x^{v / \phi} .
\end{aligned}
$$

Equation (2.2) ensures that $D$ becomes independent of $N$ at large $N$.

Equation (2.3) corresponds to the limit of no adsorption, where the size $D$ coincides with the size of a free coil in solution

$$
D(x \rightarrow 0)=N^{v} a,
$$

where $v$ is an exponent close to $3 / 5$. Since all powers of $\delta$ must drop out in (2.4) we must have $u=v / \phi$. In all what follows we shall be interested only in the limit $x \gg 1$, and write

$$
D=D(x=\infty)=a \delta^{-v / \phi}
$$

Let us now discuss the concentration profile $\phi(x)$. Our original assumption $[3,4]$ was a regular profile

$$
\phi(x)=\phi_{\mathrm{s}} v\left(\frac{x}{D}\right),
$$

where $v(0)=1$ and $v^{\prime}(0)$ were finite, and $v(y \rightarrow \infty)=0$. However, as we shall see, equation (2.6) is too restrictive ; there is a singularity in $\phi(x)$ near the wall, and we should write instead of (2.6)

$$
\phi(x)=\phi_{\mathrm{s}}\left(\frac{a}{x}\right)^{m} v\left(\frac{x}{D}\right),
$$

where $v(y)$ retains the same qualitative features, but where a new exponent $m(>0)$ appears. We call $m$ the proximal exponent. ( $m=-a$ in the notation of reference [1].)

One important consequence of (2.7) is related to the fraction $f$ of monomers which are in direct contact with the wall

$$
\begin{aligned}
& f=a \phi_{\mathrm{s}} / \int_{0}^{\infty} \phi(x) \mathrm{d} x \\
& \cong\left(\frac{a}{D}\right)^{1-m} .
\end{aligned}
$$

(1) $\phi$ should be clearly distinguished from the concentration profile $\phi(x)$. 
When $m=0$ we return to $f=a / D$ as postulated in $[3,4]$. With $m \neq 0$, we may still express the free energy per chain $F$ in terms of $f$

$$
\frac{F-F_{0}}{N k T}=-f \delta+\left(\frac{a}{D}\right)^{1 / v},
$$

where the second term is the standard confinement energy [3], but we now use equation (8) for $f$. After optimization with respect to $D$, we reach the condition

$$
\delta=\left(\frac{a}{D}\right)^{1 / v+m-1} .
$$

Comparing this result with equation (2.5) we can express $m$ in terms of $v$ and $\phi$

$$
m=1+\frac{\phi-1}{v}
$$

The assumption $m=0$ of our previous work implied a very specific value of $\phi=1-v$. However the calculations of references $[1,2]$ show that the correct value of $\phi$ is different : in three dimensions $\phi \sim 3 / 5$ giving $m \sim 1 / 3\left({ }^{2}\right)$. Thus the profile is indeed weakly singular near the wall. The $\delta$ dependence of the adsorption free energy is also interesting.

Inserting (2.10) into $(2.9)$ we find

$$
\begin{gathered}
\frac{F-F_{0}}{N k T} \sim-\delta^{q}, \\
q=\frac{1}{v(m-1)+1} .
\end{gathered}
$$

Numerically, with $m=1 / 3$ we expect $q=5 / 3$. Recently the free energy has been studied by a careful extrapolation of exact enumerations by $\mathrm{T}$. Ishinabe [5]. His result is $q=1.7 \pm 0.3$. He pointed out that this value of $q$ was not compatible with our earlier prediction $(m=0)$ leading to $q=5 / 2$. Thus there is very good agreement between the Monte Carlo data of reference [1] and the series expansions of reference [5].

\section{The many chain problem.}

For semi-dilute solutions, we expect that the singular behaviour with exponent $\boldsymbol{m}$ will persist in the proximal region. In the central and distal regions, the profile shapes remain unaltered. However, the proximal exponent does modify the boundary conditions at the surface. This in turn effects the boundary of the central domain.

In the central region, the profile is self-similar [3] and given by $x \cong \xi(\phi)$, where $\xi$ is the local correlation length

$$
\xi / a \cong \phi^{\alpha}, \quad \alpha=v /(d v-1)
$$

We propose then for the many chain case

$$
\phi(x) \cong \phi_{\mathrm{s}}\left(\frac{a}{x}\right)^{m} \tilde{v}\left(\frac{x}{D}\right)
$$

(2) The best estimate from ref. [1] is $m=0.30$. 
with a form such as :

$$
\left.\begin{array}{l}
\tilde{v}\left(\frac{x}{D}\right) \cong|D /(x+d)|^{p} \\
p+m \cong \alpha^{-1}
\end{array}\right\}
$$

in order to assure correct behaviour for $x>D$. Furthermore, insisting upon a smooth crossover between proximal and central regions yields a relationship between the surface volume fraction $\phi_{\mathrm{s}}$ and the extrapolation length [9] $D$

$$
\phi_{\mathrm{s}} \cong(a / D)^{p} .
$$

Finally, the remaining parameter $D$ is determined by optimizing the surface free energy per unit area $\gamma$, which may be written [10]

$$
\gamma-\gamma_{0}=-\gamma_{1} \phi_{\mathrm{s}}+\delta \gamma
$$

Here $\gamma_{0}$ is the interfacial energy associated with the pure solvent $\gamma_{1} a^{2}=k T \delta$, and $\delta \gamma$ is the cost in volume free energy created by the distortion of the concentration profile in the vicinity of the surface.

Following reference [6], we write in 3 dimensions :

$$
\delta \gamma \cong T \int_{0}^{\infty}[\xi(\phi)]^{-3} \mathrm{~d} x=k T / D^{2}
$$

where we have used equations (3.2), (3.3). Minimizing the interfacial energy with respect to $D$, we recover equation (1.5) for $D$. This indicates that our extension to the many chain case is plausible.

The corresponding jump in the surface energy $\Delta \gamma=\gamma-\gamma_{0}$ is given by :

$$
\Delta \gamma / \gamma_{1} \cong \delta^{p / 2-p}
$$

which is our principal result. Using $v \cong 3 / 5$ and the EKB value ( $m \cong 1 / 3$ ) for the proximal exponent, we find $p \cong 1$ and $\left|\Delta \gamma / \gamma_{1}\right| \cong \delta$. This is to be compared with our earlier scaling theory with the $m=0$ ansatz, giving $p \cong 4 / 3$ and $\left|\gamma / \gamma_{1}\right| \alpha \delta^{2}$. The jump in surface tension is linear in the surface coupling constant. (This is very similar to the $\theta$ solvent situation [11], where the proximal exponent vanishes [1].) This result, that the interfacial energy jump is only weakly dependent on the solvent quality, is consistent with recent surface tension experiments [12].

The concentration dependence of the interfacial energy may be determined by use of Gibbs relation

$$
\frac{\partial \gamma}{\partial \mu}=-\Gamma
$$

where $\mu\left(\cong k T \xi_{\mathrm{b}}^{-3} \phi_{\mathrm{b}}^{-1}\right)$ is the chemical potential and $\Gamma\left[\cong \int_{0}^{\infty} a^{-3} \phi(x) \mathrm{d} x\right]$ is the surface excess. $\Gamma$ is approximately independent of the bulk concentration $\phi_{\mathrm{b}}$ and is given by

$$
\Gamma \cong a^{-3} D\left(\frac{a}{D}\right)^{1 / \alpha}
$$

Integrating equation (3.8), we find

$$
\left(\gamma-\gamma_{0}\right) / \gamma_{1} \cong-\delta^{\sigma_{t}}+\delta^{\lambda} \phi_{\mathrm{b}}^{\tau},
$$


where each term has an undetermined numerical factor and

$$
\sigma=p /(2-p) \lambda=\frac{1-\alpha(3-p)}{\alpha(2-p)} \tau=3 \alpha-1 .
$$

In table I, we list $\sigma, \lambda, \tau$ in scaling theory for good solvents (both including and neglecting the proximal exponent), mean field theory [15] and $\theta$ solvents [11]. Systematic concentration and temperature dependence investigations of surface tensions or contact angles should provide experimental values of these exponents. Existing data $[4,11]$ are not inconsistent with these values.

Table I. - Surface tension exponents defined in equation (12). The first three columns compare scaling theories with mean field results. The fourth column gives the exponents for a mean field theory of $\theta$ solvents.

\begin{tabular}{|l|c|c|c|c|}
\hline & $\begin{array}{c}\text { Scaling } \\
m=1 / 3\end{array}$ & $\begin{array}{c}\text { Scaling } \\
m=0\end{array}$ & $\begin{array}{c}\text { Mean } \\
\text { field }\end{array}$ & $\theta$ \\
\hline$\sigma$ & 1 & 2 & 2 & $t$ \\
\hline$\lambda$ & $-2 / 3$ & $-1 / 2$ & 0 & -1 \\
\hline$\tau$ & $5 / 4$ & $5 / 4$ & 1 & 2 \\
\hline
\end{tabular}

\section{Interacting plates.}

The proximal exponent also effects the scaling predictions [13] for the force between two surfaces with adsorbed polymers. If $2 h$ is the separation of the two surfaces, and assuming irreversibly adsorbed chains incubated at large distances $\left(h \gg \xi_{\mathrm{b}}\right)$, the disjoining pressure $\Pi$ for separations in the central region $\left(D<h<\xi_{\mathrm{b}}\right)$, is universal and given by $\Pi \cong T / h^{3}$ as announced in reference [13].

However, for separations in the proximal region $(h<D)$, the results depend on $m$. We have not done a complete calculation for this case. However, we may present a simple conjecture : we assume $\Pi=\bar{\Pi}$, where $\bar{\Pi}$ is the osmotic pressure of a semi-dilute polymer solution whose concentration $\bar{\phi}$ is fixed at a value consistent with irreversible adsorption. The effective concentration $\bar{\phi}$ is given by

$$
\bar{\phi} \cong \Gamma a^{3} / h \cong(D / h)(a / D)^{1 / \alpha}
$$

The resulting disjoining pressure is then

$$
\begin{aligned}
\Pi & \cong k T / \bar{\xi}^{3} \cong\left(T / a^{3}\right) \bar{\phi}^{3 \alpha} \\
& \cong\left(k T / a^{3}\right)(D / h)^{3 \alpha}(a / D)^{3} \\
& \cong\left(k T / a^{3}\right)(a / h)^{3 \alpha} \delta^{\frac{3(1-\alpha)}{2-p}}
\end{aligned}
$$

For good solvents, $\Pi \sim h^{-9 / 4} \delta^{3 / 4}$, instead of $\Pi \sim h^{-9 / 4} \delta^{9 / 8}$, which was found for $m=0$. 


\section{Conclusions.}

In situations of weak adsorption (large $D$, small $\delta$ ) the influence of the proximal exponent $m$ can be important. In most practical cases however, we expect an adsorption strength $\delta \sim 1$. Then the proximal region fades out : the essential part of the adsorption cloud is in the central region, and the exponent $m$ has no visible effect.

\section{Acknowledgments.}

We thank Drs Eisenriegler, Kremer and Binder for a preprint (ref. [1]), and we are pleased to acknowledge helpful discussions with C. Taupin and T. Witten.

\section{References}

[1] Eisenriegler, E., Kremer, K. and Binder, K., J. Chem. Phys. 77 (1982) 6296.

[2] Diehl, H. and Dietrich, S., Phys. Rev. B 24 (1981) 2878.

[3] De Gennes, P. G., Macromolecules 14 (1981) 1637.

[4] Ober, R., Paz, L., Taupin, C., Pincus, P. and Boileau, S., Macromolecules, to be published.

[5] Ishinabe, T., J. Chem. Phys. 76 (1982) 5589.

[6] For good solvents $\xi \sim \phi^{-3 / 4}$. See DE Gennes, P. G., Scaling Concepts in Polymer Physics (Cornell University Press, Ithaca) 1979.

[7] Bray, A. and Moore, M., J. Phys. A 10 (1977) 1927.

[8] $v \cong 3 / 5$ for flexible chains in good solvents; $v=1 / 2$ in $\theta$ solvents.

[9] The scaling arguments that we use here are unable to yield numerical prefactors. Our considerations are thus restricted to dependences on the physical parameters.

[10] We make two assumptions with regard to the form of the polymer-surface interaction : (i) $\phi_{\mathrm{s}} \ll 1$, so that higher-order terms in $\phi_{s}^{2}, \phi_{\mathrm{s}}^{3}$ may be neglected to reduce the number of parameters; (ii) we assume only contact interactions; i.e., we neglect the $x^{-3}$ Van der Waals energy. This effect of the long-range forces is essentially a renormalization [3] of $\gamma_{1}$.

[11] di Meglio, J. M., Ober, R., Paz, L., Taupin, C., Pincus, P. and Boileau, S., to be published.

[12] TAUPIN, C., private communication.

[13] De Gennes, P. G., Macromolecules 15 (1982) 492. 